TRANSACTIONS OF THE

AMERICAN MATHEMATICAL SOCIETY

Volume 184, October 1973

\title{
THE PERIODS OF EICHLER INTEGRALS FOR KLEINIAN GROUPS
}

BY

\section{HIROKI SATO}

ABSTRACT. We shall give period relations and inequalities for Eichler integrals for Kleinian groups $\mathbf{r}$ which have simply connected components of of the region of discontinuity of $r$. These are a generalization of those for abelian integrals. By using the period inequality, we shall give an alternate proof of a result of $\mathrm{Kra}$.

0. Introduction. Let $\Gamma$ be a nonelementary finitely generated Kleinian group, and $\Delta_{1}$ a simply connected component of the region of discontinuity $\Omega$ of $\Gamma$.

M. Eichler [4], L V. Ahlfors [2], L. Bers [3] and I. Kra [5], [6] have represented periods of Eichler integrals as polynomials of degree at most $2 q-2, q \geq$ 2 being an integer. By this method, however, period relations for Eichler integrals are very complicated even when $\Gamma$ is a Fuchsian group of the first kind (Eichler [4]). On the other hand, G. Shimura [7] has regarded the periods as column number vectors of length $2 q-1$. In his paper he gave a certain period relation for Fuchsian groups.

By using Shimura's idea, we shall give period relations and inequalities for Eichler integrals for Kleinian groups. These are a generalization of those for abelian integrals. The main results in this paper are Theorems 1 and 2.

We shall state some notations in $\$ 1$ and some lemmas in $\$ 2$. In $\S 3$ we shall prove Theorem 1 and in $\$ 4$ we shall state the period relations and inequalities, and give an alternate proof for the Kra result [5].

The author wishes to express his deep appreciation to Professor I. Kra, K. Mathumoto and K. Oikawa for encouragement and advice.

1. Notation. Throughout this paper $\Gamma$ denotes a nonelementary finitely generated Kleinian group with a simply connected component $\Delta_{1}$ of the region of discontinuity $\Omega$ of $\Gamma$. We denote by $\Lambda$ the limit set, $\lambda(z)|d z|$ the Poincaré metric on $\Omega$. Let $q \geq 1$ be an integer. Set $\Delta=\bigcup_{A \in \Gamma} A\left(\Delta_{1}\right)$. It is a well-known fact (cf. [1]) that $\Delta / \bar{\Gamma}$ is a Riemann surface which is obtained from a compact Riemann surface, denoted by $\overline{\Delta / \Gamma}$, by deleting a finite number of points. It is

Received by the editors February 1, 1972 and, in revised form, January 16, 1973. $32 \mathrm{~N} 10$.

AMS (MOS) subject classifications (1970). Primary 30A58; Secondary 10D15, 20H10,

Key words and phrases. Eichler integrals, Kleinian groups, cohomology group, potential, Beltrami coefficients, period relation and inequality.

Copyright $\odot$ 1974, American Mathematical Society 
also known that $\Delta$ is a (disconnected) covering surface of $\Delta / \Gamma$ which ramifies over only a finite number of points.

We denote by $\mathbf{R}^{n}$ and $\mathbf{C}^{n} n$-dimensional vector spaces over $\mathbf{R}$ and $\mathbf{C}$, respectively, $n \geq 0$ be ing an integer. We regard an element in $R^{n}\left(C^{n}\right)$ as a matrix with $n$ rows and 1 column. We consider an element of $\Gamma$ as a matrix $A=\left(\begin{array}{ll}a & b \\ c & d\end{array}\right)$ with $a d-b c=1$. We denote by $\mathrm{GL}(m, \mathrm{C})$ the group of $m \times m$ invertible matrices over C. Let $\left(\begin{array}{c}u \\ v\end{array}\right)$ be a vector in $C^{2}$. For each $n=2 q-2$, we denote by $\left(\begin{array}{c}u \\ v\end{array}\right)^{n}$ the vector in $\mathrm{C}^{n+1}$ whose components are $u^{n}, u^{n-1}, \cdots, u v^{n-1}, v^{n}$, where $(\underset{v}{u})^{0}=1$. For $A \in \Gamma$ we set $\left(\begin{array}{c}u_{v_{A}}^{A} \\ )\end{array}\right)=A\left(\begin{array}{c}u \\ v\end{array}\right)$ and define $M(A) \in \mathrm{GL}(n+1, \mathrm{C})$ by

$$
\left(\begin{array}{l}
u_{A} \\
v_{A}
\end{array}\right)^{n}=M(A)\left(\begin{array}{l}
u \\
v
\end{array}\right)^{n}
$$

The following is due to Ahlfors [1]. Let $\Delta / \Gamma=S-\{p\}$ where $S$ is a Riemann surface and $p \in S$. If there is a punctured neighborhood $M(p)$ of $p$ such that $\pi$ is unramified over $M(p)$, then there exists a parabolic transformation $A \epsilon$ $\Gamma$ with fixed point $s \in \Lambda$, and there is a Möbius transformation $B$ with the following properties: (1) $B(\infty)=s$ and $B^{-1} A B(z)=z+1, z \in \mathrm{C}$, (2) $B^{-1}\left(\Delta_{1}\right)$ contains a half-plane $U_{B-1}{ }_{B}=\{z \in \mathrm{C} \mid \operatorname{Im} z>c\}$, for some $c>0$, (3) two points $z_{1}$ and $z_{2}$ of $B\left(U_{B-1}{ }^{-1} B\right)$ are equivalent under $\Gamma$ if and only if $z_{2}=A^{m}\left(z_{1}\right)$ for some integer $m$, and (4) the image of $B\left(U_{B-1}{ }_{B}\right)$ under $\pi$ is a deleted neighborhood of $p$ homeomorphic to a punctured disk. We call $W_{A}=B\{z \in \mathbf{C} \mid 0 \leq \operatorname{Re} z$ $<1, \operatorname{Im} z>c\}$ a cusped region belonging to $p$.

$A$ mapping $\chi: \Gamma \rightarrow C^{2 q-1}$ is called a cocycle if $\chi_{A B}={ }^{t} M(B) \chi_{A}+\chi_{B}$ for $A, B \in \Gamma$, where ${ }^{t} M(B)$ is the transposed matrix of $M(B)$. A cocycle $\chi: \Gamma \rightarrow$ $\mathrm{C}^{2 q-1}$ is called a coboundary if there exists $V \in \mathrm{C}^{2 q-1}$ such that $\chi_{A}={ }^{t} M(A) V$ $-V$ for any $\chi_{A} \in \mathbf{C}^{2 q-1}, A \in \Gamma$. Then the first cohomology group $H^{1}\left(\Gamma, \mathbf{C}^{2 q-1}, M\right)$ is the space of cocycles factored by the space of coboundaries. A cohomology class $P \in H^{1}\left(\Gamma, \mathrm{C}^{2 q-1}, M\right)$ is called $\Delta$-parabolic if, for every parabolic transformation $B \in \Gamma$ corresponding to a puncture on $\Delta / \Gamma$, there is a $V \in \mathrm{C}^{2 q-1}$ such that $P_{B}={ }^{t} M(B) V-V$ for some (and hence every) cocycle that represents $P$. The space of $\Delta$-parabolic cohomology class is denoted by $P H_{\Delta}^{1}\left(\Gamma, \mathrm{C}^{2 q-1}, M\right)$.

For an $m \times n$ matrix $N=\left(a_{i j}\right) \quad(i=1,2, \cdots, n ; j=1,2, \cdots, m)$ matrices $\bar{N}$ and $\tilde{N}$ are defined by $\bar{N}=\left(\bar{a}_{i j}^{i j}\right)$ and $\tilde{N}=\left(a_{m-i+1 n-j+1}\right)$, respectively, where $\bar{a}_{i j}$ is the complex conjugate of $a_{i j}$.

A holomorphic function $\phi$ on $\Delta$ is called an automorphic form of weight $(-2 q)$ on $\Delta, q \geq 1$, if $\phi(A z) A^{\prime}(z)^{q}=\phi(z)$ for all $A \in \Gamma$. For $q \geq 2$ an automorphic form $\phi$ of weight $(-2 q)$ on $\Delta$ is called integrable if

$$
\iint_{\Delta / \mathbf{r}} \lambda(z)^{2-q}|\phi(z)| d x d y<\infty .
$$


We denote the Banach space of integrable automorphic forms on $\Delta$ by $A_{q}(\Delta, \Gamma)$. The form $\phi$ is called bounded if

$$
\sup \left\{\lambda(z)^{-q}|\phi(z)| \mid z \in \Delta\right\}<\infty .
$$

The Banach space of bounded automorphic forms on $\Delta$ is denoted by $B_{q}(\Delta, \Gamma)$. For $\phi \in A_{q}(\Delta, \Gamma)$ and $\psi \in B_{q}(\Delta, \Gamma)$, we define the Petersson inner product by

$$
(\phi, \psi)=\iint_{\Delta / \Gamma} \lambda(z)^{2-2 q} \phi(z) \overline{\psi(z)} d x d y, \quad q \geq 2 .
$$

For $q=1$ we shall interpret $A_{1}(\Delta, \Gamma)$ and $B_{1}(\Delta, \Gamma)$ as the Hilbert space of square integrable automorphic forms of weight $(-2)$ with inner product defined by

$$
(\phi, \psi)=\iint_{\Delta / \Gamma} \phi(z) \overline{\psi(z)} d x d y .
$$

A holomorphic function $E$ on $\Delta$ is called a holomorphic Eichler integral of order $(1-q)$ on $\Delta$ if $E(A z) A^{\prime}(z)^{1-q}-E(z) \in \Pi_{2 q-2}$ on $\Delta$, for all $A \in \Gamma$, where $\Pi_{2 q-2}$ is the vector space of polynomials of degree at most $2 q-2$. We shall say that an Eichler integral $E$ of order $1-q$ is bounded if $\phi=D^{2 q-1} E \in B_{q}(\Delta, \Gamma)$, where $D$ means differentiation with respect to $z$. The projection of $\phi$ to $\Delta / \Gamma$ is then a meromorphic $q$-differential on $\overline{\Delta / \Gamma}$ with order $\geq-(q-1)$ at the punctures on $\Delta / \Gamma$. An Eichler integral $E$ on $\Delta$ is called quasi-bounded if the projection of $D^{2 q-1} E$ to $\Delta / \Gamma$ can be extended as a meromorphic $q$-differential to $\overline{\Delta / \Gamma}$ whose order at a puncture is $\geq-q$. The space of bounded Eichler integrals modulo $\Pi_{2 q-2}$ will be denoted by $P E_{1-q}(\Delta, \Gamma)$. Similarly $E_{1-q}(\Delta, \Gamma)$ denotes the space of quasi-bounded Eichler integrals modulo $\Pi_{2 q-2}$. set

Let $f \in E_{1-q}(\Delta, \Gamma)$ and $E$ a representative of $f$ and set $D^{2 q-1} E=\phi$. We

$$
f_{n-j}(z)=\sum_{k=0}^{j}\left((-1)^{k} j ! /(j-k) !\right) z^{j-k} D^{2 q-2-k} E(z)
$$

and set

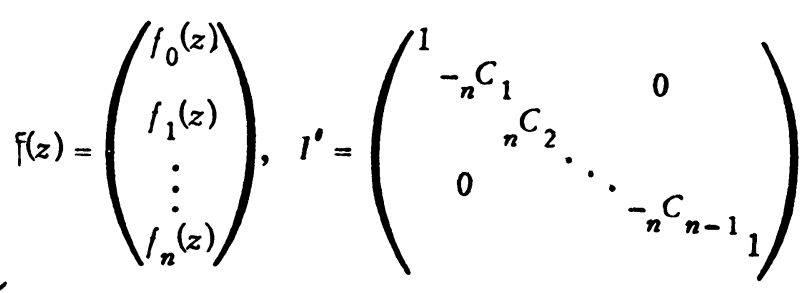

$\mathcal{F}(z)=I^{\prime} \mathrm{F}(z)$ and $\omega(z)=\phi(z)(z)^{n} d z$, where ${ }_{n} C_{i}=n ! /(n-1) ! i$. We call $f(z)$ and $\mathfrak{F}(z)$ column function vectors of length $n+1$ associated with $E$. For each $A \in \Gamma$ we define $X_{A}$ and $P_{A}$ by 


$$
X_{A}=f(A z)-M(A) f(z)
$$

and

$$
P_{A}={ }^{t} M(A) \mathfrak{F}(A z)-\mathfrak{F}(z)
$$

and denote them by $\mathrm{pd}_{A}(f)$ and $\operatorname{pd}_{A}(\mathfrak{F})$, respectively. We call $X_{A}$ and $P_{A}$ periods of $F$ and $\mathfrak{F}$ for $A \in \Gamma$, respectively. The mapping $A \mapsto P_{A}$ satisfies $P_{A B}$ $={ }^{t} M(B) P_{A}+P_{B}$ for any $A, B \in \Gamma$, as is easily seen. Then a cohomology class is defined, which depends only on $f$ and not $E$. We define by $E_{1-q}(\Delta, \Gamma, M)$ the space of all $F(z)$ modulo $C^{2 q-1}$. Similarly we define $P E_{1-q}(\Delta, \Gamma, M)$. Thus by the obvious way we may define a mapping

$$
a: E_{1-q}(\Delta, \Gamma, M) \rightarrow H^{1}\left(\Gamma, \mathrm{C}^{2 q-1}, M\right)
$$

and we know that $a\left(P E_{1-q}(\Delta, \Gamma, M)\right) \subset P H^{1}\left(\Gamma, C^{2 q-1}, M\right)$ by the method similar to that of $\mathrm{Kra}$ [6]. call

If $a_{1}, a_{2}, \cdots, a_{2 q-1}$ are distinct points in $\Lambda$, and $\psi \in B_{q}(\Delta, \Gamma)$, then we

$$
\frac{\left(z-a_{1}\right) \cdots\left(z-a_{2 q-1}\right)}{2 \pi i} \iint_{\mathbb{Q}} \frac{\lambda(\zeta)^{2-2 a} \overline{\psi(\zeta)} d \zeta \wedge d \bar{\zeta}}{(\zeta-z)\left(\zeta-a_{1}\right) \cdots\left(\zeta-a_{2 q-1}\right)},
$$

$z \in \mathrm{C}, q \geq 2$, a potential for $\psi$, and denote it by $\operatorname{Pot}(\psi)$. For $A \in \Gamma$, we define a period of potential of $\operatorname{Pot}(\psi)$ by setting

$$
\operatorname{pd}_{A} \operatorname{Pot}(\psi)(z)=\operatorname{Pot}(\psi)(A z) A^{\prime}(z)^{1-q}-\operatorname{Pot}(\psi)(z), \quad z \in \mathbf{C} .
$$

It is easily seen that $\operatorname{Pot}(\psi) \mid \Omega-\Delta \in P E_{1-q}(\Omega-\Delta, \Gamma)$ for $\psi \in B_{q}(\Delta, \Gamma)$. We set

$$
g_{n-j}(z)=\sum_{k=0}^{j}\left((-1)^{k} j ! /(j-k) !\right) z^{j-k} D^{2 q-2-k} \operatorname{Pot}(\psi)(z), \quad z \in \Omega-\Lambda .
$$

We set

$$
g(z)=\left(\begin{array}{c}
g_{0}(z) \\
g_{1}(z) \\
\vdots \\
g_{n}(z)
\end{array}\right)
$$

and set $B(z)=I^{\prime} g(z)$. We call $g(z)$ and $B(z)$ column function vectors of length $n+1$ associated with $\operatorname{Pot}(\psi)$.

For each $A \in \Gamma$, we define $Y_{A}$ and $Q_{A}$ by 


$$
Y_{A}=g(A z)-M(A) g(z), \quad z \in \Omega-\Delta,
$$

and

$$
Q_{A}={ }^{t} M(A) \&(A z)-B(z), \quad z \in \Omega-\Delta,
$$

and denote them by $\mathrm{pd}_{A}(\mathrm{~g})$ and $\mathrm{pd}(8)$, respectively. We call $Y_{A}$ and $Q_{A}$ periods of $g$ and $B$ for $A \in \Gamma$, respectively. The mapping $A \mapsto Q_{A}$ satisfies $Q_{A B}$ $={ }^{t} M(B) Q_{A}+Q_{B}$, for any $A, B \in \Gamma$, as is easily seen. Then a cohomology class is defined, which depends only on $\psi$. The definition of $Y_{A}, Q_{A}$, etc., applies to the case $\Omega-\Delta \neq \varnothing$. These functions for the remaining case will be defined in the remark after Lemma 4 . Similarly as above we define

$$
\beta^{*}: B_{q}(\Delta, \Gamma) \rightarrow H^{1}\left(\Gamma, \mathrm{C}^{2 q-1}, M\right) .
$$

Let $\Gamma_{1}$ be a subgroup of $\Gamma$ which keeps $\Delta_{1}$ invariant, and let $b$ be a conformal mapping from $\Delta_{1}$ on the upper half-plane $U$. Set $\Gamma_{1}^{\prime}=b \Gamma_{1} b^{-1}$. Then $\Gamma_{1}^{\prime}$ is a fuchsian group of the first kind. Let $\omega_{0}$ be the fundamental region defined by Shimura [7] for $\Gamma_{1}^{\prime}$ in $U$, then we let its boundary consist of sides $E_{A_{1}^{\prime}}, E_{B_{1}^{\prime}}$, $-A_{1}^{\prime}\left(E_{A_{1}^{\prime}}\right),-B_{1}^{\prime-1}\left(E_{B_{1}^{\prime}}\right), \cdots, E_{A_{g}^{\prime}}, E_{B_{g}^{\prime}},-A_{g}^{\prime}\left(E_{A_{g}^{\prime}}\right),-B_{g}^{\prime-1}\left(E_{B_{g}^{\prime}}\right), E_{C_{1}^{\prime}}$,

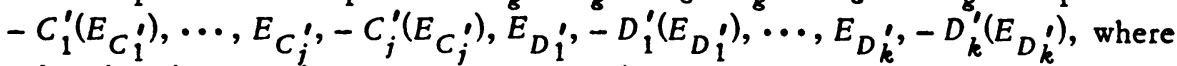
$A_{\lambda}^{\prime}, B_{\lambda}^{\prime}, C_{\mu}^{\prime}$ and $D_{\nu}^{\prime}$ are generators of $\Gamma_{1}^{\prime}$ with relations $D_{k} \cdots D_{1} C_{j} \cdots$ $C_{1} \Pi_{\lambda=1}^{g} B_{\lambda}^{\prime-1} A_{\lambda}^{\prime-1} B_{\lambda}^{\prime} A_{\lambda}^{\prime}=1$ and $C_{\mu}^{\prime e} \mu=1(\mu=1,2, \cdots, j)$. If we set $A_{\lambda}=$ $b^{-1} A_{\lambda}^{\prime} b, B_{\lambda}=b^{-1} B_{\lambda}^{\prime} h, C_{\mu}=b^{-1} C_{\mu}^{\prime} b$ and $D_{\nu}=b^{-1} D_{\nu}^{\prime} b(\lambda=1, \cdots, g ; \mu=1, \cdots$, $j ; \nu=1, \cdots, k)$, then $\Gamma_{1}$ is generated by $A_{\lambda}, B_{\lambda}, C_{\mu}$ and $D_{\nu}$ with relations $D_{k}$ $\because D_{1} C_{j} \cdots C_{1} \Pi_{\lambda=1}^{g} B_{\lambda}^{-1} A_{\lambda}^{-1} B_{\lambda} A_{\lambda}=1$ and $C_{\mu}^{e}=1(\mu=1, \cdots, j)$. We set $\omega_{0}=$ $b^{-1}\left(\omega_{0}^{\prime}\right)$ and set $E_{A_{\lambda}}=b^{-1}\left(E_{A_{\lambda}^{\prime}}\right), E_{B_{\lambda}}=b^{-1}\left(E_{B_{\lambda}}\right), E_{C_{\mu}}=b^{-1}\left(E_{\left.C_{\mu}^{\prime}\right)}\right.$ and $E_{D_{\nu}}$ $=b^{-1}\left(E_{D_{\nu}}\right)$. Then we easily see that $\omega_{0}$ is a fundamental region for $\Gamma_{1}$ in $\Delta_{1}$ whose boundary consists of $E_{A_{1}}, E_{B_{1}},-A_{1}\left(E_{A_{1}}\right),-B_{1}^{-1}\left(E_{B_{1}}\right), \cdots, E_{A_{g}}, E_{B_{g}}$, $-A_{8}\left(E_{A_{g}}\right),-B_{g}^{-1}\left(E_{B_{g}}\right), E_{C_{1}},-C_{1}\left(E_{C_{1}}\right), \ldots, E_{j},-C_{j}\left(E_{C_{j}}\right), E_{D_{1}},-D_{1}\left(E_{D_{1}}\right)^{B^{\prime}}$, $\cdots, E_{D_{k}},-D_{k}\left(E_{D_{k}}\right)$, since $-A_{\lambda}\left(E_{A_{\lambda}}\right)=b^{-1}\left(A_{\lambda}^{\prime}\left(E_{A_{\lambda}^{\prime}}\right)\right),-B_{\lambda}\left(E_{B_{\lambda}}\right)=b^{-1}\left(-B_{\lambda}^{\prime}\left(E_{B_{\lambda}^{\prime}}\right)\right)$, $-C_{\mu}\left(E_{C_{\mu}}\right)=b^{-1}\left(-C_{\mu}^{\prime}\left(E_{C_{\mu}^{\prime}}\right)\right)$ and $-D_{\nu}\left(E_{D_{\nu}}\right)=b^{-1}\left(-D_{\nu}^{\prime}\left(E_{D_{\nu}^{\prime}}\right)\right)$. Since $b$ is a conformal mapping, the sides of $\omega_{0}$ consist of piecewise analytic arcs. We set $S_{\lambda}=B_{\lambda}^{-1} A_{\lambda}^{-1} B_{\lambda} A_{\lambda}$ and $T_{\lambda}=S_{\lambda} \cdots S_{1}(\lambda=1, \cdots, g)$.

2. Lemmas. In this section we state some lemmas which are necessary to prove the subsequent theorems. Many of the properties in Lemmas 2, 3, 4 and 5 below can be sumarized by saying that there is an isomorphism $\Pi_{2 q-2} \rightarrow C^{2 q-1}$ which commutes with the action of $\Gamma$. However we shall state them for the sake of later use. For each $A=\left(\begin{array}{ll}a & b \\ c & d\end{array}\right) \in \Gamma$, we denote by $A(z)=(a z+b) /(c z+d)$. We set $n=2 q-2$ once and for all. 
Lemma 1. For $A \in \Gamma$

$$
\left.M(A)=I^{\prime-1}(\overbrace{}^{t} M(A))\right)^{-1} l^{\prime}
$$

Proof. We set $A=\left(\begin{array}{ll}a & b \\ c & d\end{array}\right), a d-b c=1$. The $(k, l)$ element of $M(A)$ is

$$
\sum_{i+j=l-1} 2 q-1-k C_{i k-1} C_{j} a^{2 q-k-i-1} c^{k-1-j} b^{i} d^{j}
$$

The $(k, l)$ element of $M(A)$ is the $(l, k)$ element of ${ }^{t} M(A)$, which is the $(2 q-l$, $2 q-k)$ element of ${ }^{t} M(A)$. Hence the $(2 q-l, 2 q-k)$ element of ${ }^{t} M(A) I^{\prime}$ is

(3) $(-1)^{2 q-k-1}{ }_{2 q-2} C_{2 q-k-1} \sum_{i+j=l-1} 2 q-1-k C_{i k-1} C_{j} a^{2 q-1-k-i} c^{k-1-i} b^{i} d^{j}$.

On the other hand the $(2 q-l, 2 q-k)$ element of $I^{\prime} M\left(A^{-1}\right)$ is

$$
{ }^{(-1)^{2 q-1-1}}{ }_{2 q-2} C_{2 q-1-1} \sum_{i+j=2 q-1-k}{ }^{l-1} C_{i} 2 q-1-1 C_{l} d^{l-1-i}(-c)^{2 q-1-1-j}(-b)^{i} a^{j} \text {. }
$$

We easily see that (3) and (4) are the same, that is,

$$
\left(\widetilde{t^{\prime} M(A)}\right) I^{\prime}=I^{\prime}, M\left(A^{-1}\right) \text {. }
$$

For the proof of some properties in Lemmas 2 through 4 below, see Shimura [7].

Lemma 2. For $A, B \in \Gamma$,

(1) $\left({ }_{1}^{A z}\right)^{n} A^{\prime}(z)^{1-q}=M(A)(\underset{1}{1})^{n}$,

(2) $M(A B)=M(A) M(B)$,

(3) $M\left(A^{-1}\right)=M(A)^{-1}$.

Lemma 3. Let $f \in E_{1-q}(\Delta, \Gamma)$ and $E$ a representative of fo and $\psi \in B_{q}(\Delta, \Gamma)$. Let $f(z), \omega(z), g(z), X_{A}, Y_{A}, P_{A}$ and $Q_{A}$ be defined as in $\S_{1}$ with respect to $E$ and $\psi$. Then, for $A, B \in \Gamma$,

(1) $\omega(A z)=M(A) \omega(z)$,

(2) $d f(z)=\omega(z)$,

(3) $E(z)=(1 / n !)^{t} \mathcal{f}(z) I^{\prime}\left(\frac{1}{z}\right)^{n}, z \in \Delta$,

(4) $E(A z) A^{\prime}(z)^{1-q}-E(z)=(1 / n !){ }^{t} P_{A}\left({ }_{1}\right)^{n}, z \in \Delta$,

(5) $X_{A B}=X_{A}+M(A) X_{B}$,

(6) $\operatorname{Pot}(\psi)(z)=(1 / n !){ }^{t} g(z) I^{\prime}\left({ }_{z}^{1}\right)^{n}, z \in \mathbf{C}$,

(7) $\operatorname{Pot}(\psi)(A z) A^{\prime}(z)^{1-q}-\operatorname{Pot}(\psi)(z)=(1 / n !){ }^{t} Q_{A}\left(\frac{z}{1}\right)^{n}, z \in \Omega-\Delta$,

(8) $Y_{A B}=Y_{A}+M(A) Y_{B}$,

(9) $P_{A}=I^{\prime} M(A)-1 \widetilde{X}_{A}, X_{A}=M(A) I^{1-1} \widetilde{P}_{A}$ ?

(10) $Q_{A}=I \stackrel{M(A)}{-1} \widetilde{Y}_{A}, \dot{Y}_{A}=M(A) I^{1}-1 \widetilde{Q}_{A}$.

By (3) of Lemma 3 we have 
Lemma 4. For each $A \in \Gamma, X_{A}, P_{A}, Y_{A}$ and $Q_{A}$ are all number vectors of length $2 q-1$.

This means that

$$
\operatorname{Pot}(\psi)(A z) A^{\prime}(z)^{1-q}-\operatorname{Pot}(\psi)(z)<(1 / n !)^{t} Q_{A}\left(\begin{array}{l}
z \\
1
\end{array}\right)^{n}
$$

for $z \in \mathbf{C}$ and $A \in \Gamma$.

Remark. The above $Q_{A}$ is defined in the case of $\Omega \neq \Delta$. However, since $\operatorname{Pot}(\psi)(A z) A^{\prime}(z)^{1-q}-\operatorname{Pot}(\psi)(z)=v_{A}(z), v_{A} \in \Pi_{2 q-2}, A \in \Gamma, z \in \mathbf{C}$, we may define $Q_{A}^{\prime}$ by $v(A)=(1 / n !)^{t} Q_{A}^{\prime}\left(\begin{array}{c}z \\ 1\end{array}\right)^{n}$ with $Q_{A}^{\prime} \in \mathrm{C}^{2 q-1}$. Then we easily see that $Q_{A B}^{\prime}={ }^{t} M(B) Q_{A}^{\prime}+Q_{B^{\prime}}^{\prime} A, B \in \Gamma$. We set $Y_{A}^{\prime}=M(A) I^{\prime-1}{ }^{\prime}{ }_{A}^{\prime}$. Hereafter we take $Q_{A}$ to be $Q_{A}$ and $Y_{A}$ to be $Y_{A}^{\prime}$, and note that these definitions agree with previous ones and are valid in the case $\Omega=\Delta$ as well.

Noting the fact pointed out in the first part of this section, we have the following from Kra's decomposition theorem [5], [6].

Lemma 5. (1) $H^{1}\left(\Gamma, \mathrm{C}^{2 q-1}, M\right)=\alpha\left(E_{1-q}(\Delta, \Gamma, M)+\beta^{*}\left(B_{q}(\Delta, \Gamma)\right)\right.$;

(2) $\beta^{*}\left(B_{q}(\Delta, \Gamma)\right) \subset P H_{\Delta}^{1}\left(\Gamma, C^{2 q-1}, M\right)$.

3. The main theorem.

Theorem 1. Let $\Gamma$ be a nonelementary finitely generated Kleinian group and $\Delta_{1}$ a simply connected component of $\Omega$ and set $\Delta=\bigcup_{A \in \Gamma} A\left(\Delta_{1}\right)$. Let $f \epsilon$ $E_{1-q}(\Delta, \Gamma), E$ an arbitrary representative of $f$ and set $D ? q-1 E=\phi, q \geq 2$. Let $\psi \in B_{q}(\Delta, \Gamma)$. Let $F(z)$ and $g(z)$ be column function vectors (1) and (2) associated with $E$ and Pot $(\psi)$, respectively, and set $B(z)=I^{\prime} g(z)$. Let $\operatorname{pd}_{A} f(z)=X_{A}$ and $\operatorname{pd}_{A} \mathbb{S}(z)=Q_{A}$ for each $A \in \Gamma$. Then

$$
\begin{aligned}
& \operatorname{2in} !(\phi, \psi)=\sum_{\lambda=1}^{8}{ }^{\iota} Q_{A_{\lambda}}\left[X_{A_{\lambda}^{-1} B_{\lambda} A_{\lambda} T_{\lambda-1}}-X_{T_{\lambda-1}}\right] \\
& +\sum_{\lambda=1}^{g}{ }^{t} Q_{B_{\lambda}^{-1}}\left[X_{B_{\lambda} A_{\lambda} T_{\lambda-1}}-X_{A_{\lambda}^{-1} B_{\lambda} A_{\lambda} T_{\lambda-1}}\right] \\
& +\sum_{\mu=1}^{j} Q_{C_{\mu}}\left[e_{\mu}^{-1} \sum_{m=1}^{e_{\mu}-1} X_{C_{\mu}}-X_{C_{\mu-1} \ldots C_{1} T_{g}}\right]
\end{aligned}
$$

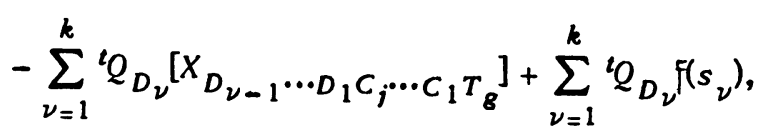

where $s_{\nu}$ is a cusp point of $D_{\nu}(\nu=1,2, \cdots, k)$. 
Proof. By (7) of Lemma 3 and Lemma 4, for every $A \in \Gamma$,

$$
\operatorname{Pot}(\psi)(A z) A^{\prime}(z)^{1-q}-\operatorname{Pot}(\psi)(z)=(1 / n !)^{t} Q_{A}\left(\begin{array}{l}
z \\
1
\end{array}\right)^{n}, \quad z \in \mathbf{C} .
$$

Let $\eta(z)$ be a $C^{\infty}$-function on $\Delta$ defined by $\mathrm{Kra}$ [5], [6], that is, (1) $0 \leq \eta \leq 1$;

(2) for each $z \in \Delta$, there is a neighborhood $U(z)$ of $z$ and a finite subset $J$ of $\Gamma$ such that $\eta(A(U(z)))=0$ for each $A \notin J$; (3) $\Sigma_{\gamma \in \Gamma} \eta(y z)=1, z \in \Delta$; and (4) if $U_{A} \in \omega_{0}$ is a cusped region belonging to a puncture on $\Delta / \Gamma$ and $A$ is the corresponding transformation, then $\eta \mid B\left(U_{A}\right)=0$ all $B \in \Gamma-\{1, A\}$.

We set

$$
\Theta(z)=\left(\begin{array}{c}
\theta_{0}(z) \\
\theta_{1}(z) \\
\vdots \\
\theta_{n}(z)
\end{array}\right)=-\frac{1}{n !} \sum_{\gamma \in \Gamma} \eta(\gamma z) Q_{\gamma}+\frac{1}{n !} \sum_{\nu=1}^{k} \chi_{\nu}(z)\left\{\sum_{\gamma \in \Gamma} \eta(\gamma z){ }^{t} M(\gamma) V_{\nu}\right\}
$$

where $V_{\nu}$ is defined by ${ }^{t} M\left(D_{\nu}\right) V_{\nu}-V_{\nu}=P_{D_{\nu}}(\nu=1, \cdots, k)$ and we let $\chi_{\nu} \epsilon$ $C^{\infty}(\Delta, \Gamma)$ be such that $0 \leq \chi_{\nu} \leq 1, \chi_{\nu}=1$ in $U_{D_{\nu}}$ and $\chi_{\nu}=0$ in $\bigcup_{i \in \nu} U_{D_{i}}$ $(\nu=1, \cdots, k)$. Then

$$
\begin{aligned}
{ }^{t} M(A) \Theta(A z)-\Theta(z) \\
=-\frac{1}{n !}{ }^{t} M(A) \sum_{\gamma \in \Gamma} \eta(\gamma A z) Q_{\gamma}+\frac{1}{n !}{ }^{t} M(A) \sum_{\nu=1}^{k} \chi_{\nu}(A z)\left(\sum_{\gamma \in \Gamma} \eta(\gamma A z){ }^{t} M(\gamma) V_{\nu}\right) \\
\quad+\frac{1}{n !} \sum_{\gamma \in \Gamma} \eta(\gamma z) Q_{\gamma}-\frac{1}{n !} \sum_{\nu=1}^{k} \chi_{\nu}(z)\left(\sum_{\gamma \in \Gamma} \eta(\gamma z){ }^{t} M(\gamma) V_{\nu}\right) \\
=-\frac{1}{n !} \sum_{\gamma \in \Gamma} \eta(\gamma A z)\left(Q_{\gamma A}-Q_{A}\right)+\sum_{\gamma \in \Gamma} \frac{1}{n !} \eta(\gamma z) Q_{\gamma} \\
\quad+\frac{1}{n !} \sum_{\nu=1}^{k} \chi_{\nu}(z)\left(\sum_{\gamma \in \Gamma} \eta\left(\gamma_{i} A z\right){ }^{t} M(\gamma A) V_{\nu}\right) \\
\quad-\frac{1}{n !} \sum_{\nu=1}^{k} \chi_{\nu}(z)\left(\sum_{\gamma \in \Gamma} \eta(\gamma z){ }^{t} M(\gamma) V_{\nu}\right) \\
=\frac{1}{n !} Q_{A},
\end{aligned}
$$

for each $A \in \Gamma$. 
We set

$$
\mu(z)=\left(\begin{array}{c}
\mu_{0}(z) \\
\mu_{1}(z) \\
\vdots \\
\mu_{n}(z)
\end{array}\right)=\left(\begin{array}{c}
\partial \theta_{0}(z) / \partial \bar{z} \\
\partial \theta_{1}(z) / \partial \bar{z} \\
\vdots \\
\partial \theta_{n}(z) / \partial \bar{z}
\end{array}\right)
$$

Then we easily see that, for $A \in \Gamma$,

$$
{ }^{t} M(A) \mu(A z) \overline{A^{\prime}(z)}=\mu(z), \quad z \in \Delta
$$

Thus for $\phi=D^{2 q-1} E$,

$$
\iint_{\Delta / \Gamma} \phi(z){ }^{i} \mu(z)\left(\begin{array}{c}
z \\
1
\end{array}\right)^{n} d z \wedge d \bar{z}
$$

is well defined.

Next we show that

$$
\iint_{\omega_{0}} \phi(z)^{i} \mu(z)\left(\begin{array}{c}
z \\
1
\end{array}\right)^{n} d z \wedge d \bar{z}-\iint_{\omega_{0}} \phi(z) \lambda(z)^{2-2 q} \overline{\psi(z)} d z \wedge d \bar{z}=0 .
$$

For, since for $A \in \Gamma$,

$$
{ }^{t} \Theta(A z)\left(\begin{array}{c}
A z \\
1
\end{array}\right)^{n} A^{\prime}(z)^{1-q}-{ }^{t} \Theta(z)\left(\begin{array}{c}
z \\
1
\end{array}\right)^{n}=\frac{1}{n !}{ }^{t} Q_{A}\left(\begin{array}{c}
z \\
1
\end{array}\right)^{n}, \quad z \in \Delta,
$$

by (5) we have

$$
\left\{{ }^{t} \Theta(A z)\left(\begin{array}{c}
A z \\
1
\end{array}\right)^{n}-\operatorname{Pot}(\psi)(A z)\right\} A^{\prime}(z)^{1-q}={ }^{t} \Theta(z)\left(\begin{array}{c}
z \\
1
\end{array}\right)^{n}-\operatorname{Pot}(\psi)(z),
$$

$z \in \Delta$. By us ing Stokes' the orem after Bers' trick [3], (6) is equal to

$$
\int_{\partial \omega_{0}}\left\{t_{\Theta}(z)\left(\begin{array}{l}
z \\
1
\end{array}\right)^{n}-\operatorname{Pot}(\psi)(z)\right\} \phi(z) d z .
$$

This is equal to zero, in fact, since its integrals along two identified sides cancel each other and, therefore, (8) is equal to zero, that is,

$$
-2 i(\phi, \psi)=\iint_{\omega_{0}} \phi(z)^{t} \mu(z)\left(\begin{array}{l}
z \\
1
\end{array}\right)^{n} d z \wedge d \bar{z} .
$$


448

HIROKI SA TO

On the other hand,

$$
\iint_{\omega_{0}} t \mu(z) \phi(z)\left(\begin{array}{c}
z \\
1
\end{array}\right)^{n} d z \wedge d \bar{z}=\int_{\partial \omega_{0}} t^{t} \Theta(z)\left(\begin{array}{l}
z \\
1
\end{array}\right)^{n} \phi(z) d z=\int_{\partial \omega_{0}} \imath \Theta(z) \omega(z),
$$

where $\omega(z)=\phi(z)(z){ }_{1}^{n} d z$. Then

$$
\begin{aligned}
& \int_{\partial \omega_{0}}{ }^{t} \Theta(z) \omega(z)=\int_{\partial \omega_{0}}{ }^{t} \Theta(z) d F(z) \\
& =\sum_{\lambda=1}^{8}\left(\int_{E_{A_{\lambda}}}{ }^{t} \Theta(z) d F(z)-\int_{A_{\lambda}\left(E_{A_{\lambda}}\right)^{t} \Theta(z) d F(z)}\right. \\
& +\sum_{\lambda=1}^{8}\left(\int_{E_{B_{\lambda}}}{ }^{t} \Theta(z) d F(z)-\int_{\left.B_{\lambda}^{-1}\left(E_{B_{\lambda}}\right)^{t} \Theta(z) d F(z)\right)}\right.
\end{aligned}
$$

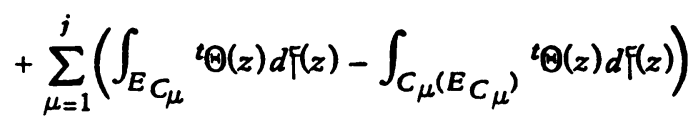

$$
\begin{aligned}
& +\sum_{\nu=1}^{k}\left(\int_{E_{D_{\nu}}}{ }^{t} \Theta(z) d f(z)-\int_{D_{\nu}\left(E_{D_{\nu}}\right)}{ }^{t} \Theta(z) d f(z)\right) .
\end{aligned}
$$

Now for any element $A$ of $\left\{A_{\lambda}, B_{\lambda}^{-1}, C_{\mu^{\prime}} D_{\nu}(\lambda=1, \cdots, g ; \mu=1, \cdots, j ; \nu=1\right.$, $\cdots, k)$,

$$
\begin{aligned}
& \int_{A\left(E_{A}\right)}{ }^{t} \Theta(z) d F(z)=\int_{E_{A}}{ }^{t} \Theta(A(z)) d F(A(z)) \\
& =\int_{E_{A}}\left\{t\left(t^{t} M\left(A^{-1}\right) \Theta(z)\right)+\frac{1}{n !}{ }^{t}\left({ }^{t} M\left(A^{-1}\right) Q_{A}\right) d\left(M(A) f(z)+X_{A}\right)\right\} \\
& =\int_{E_{A}}{ }^{t} \Theta(z) d F(z)+\int_{E_{A}} \frac{1}{n !}{ }^{t} Q_{A} d F(z),
\end{aligned}
$$

so that

$$
\begin{aligned}
\int_{\partial \omega_{0}}{ }^{t} \Theta(z) \omega(z)=- & \left\{\sum_{\lambda}\left(\int_{E_{A_{\lambda}}} \frac{1}{n !}{ }^{t} Q_{A} d F(z)+\int_{E_{B_{\lambda}}} \frac{1}{n !} Q^{t} Q_{B_{\lambda}{ }^{-1}} d F(z)\right)\right. \\
& \left.+\sum_{\mu} \int_{E_{C_{\mu}}} \frac{1}{n !}^{t} Q_{C_{\mu}} d F(z)+\sum_{\nu} \int_{E_{D_{\nu}}} \frac{1}{n !}{ }^{t} Q_{D_{\nu}} d F(z)\right\} .
\end{aligned}
$$

Denote by $u_{0}$ the starting point of $E_{A_{1}}$. Then 


$$
\begin{aligned}
& \operatorname{2in} !(\phi, \psi)=\sum_{\lambda=1}^{8}{ }^{t} Q_{A_{\lambda}}\left[M\left(A_{\lambda}^{-1} B_{\lambda} A_{\lambda} T_{\lambda-1}\right)-M\left(T_{\lambda-1}\right)\right] F\left(u_{0}\right) \\
& +\sum_{\lambda=1}^{g}{ }^{t} Q_{B_{\lambda}^{-1}}\left[M\left(B_{\lambda} A_{\lambda} T_{\lambda-1}\right)-M\left(A_{\lambda}^{-1} B_{\lambda} A_{\lambda} T_{\lambda-1}\right)\right] \mathcal{F}\left(u_{0}\right) \\
& +\sum_{\mu=1}^{j}{ }^{t} Q_{C_{\mu}}\left[M\left(C_{\mu-1} \cdots C_{1} T_{8}\right)\right] \digamma\left(u_{0}\right) \\
& -\sum_{\nu=1}^{k}{ }^{t} Q_{D_{\nu}}\left[M\left(D_{\nu-1} \cdots D_{1} C_{j} \cdots C_{1} T_{g}\right)\right] \mathcal{F}\left(u_{0}\right) \\
& +\sum_{\lambda=1}^{8}{ }^{t} Q_{A_{\lambda}}\left[X_{A_{\lambda}^{-1} B_{\lambda} A_{\lambda} T_{\lambda-1}}-X_{T_{\lambda-1}}\right] \\
& +\sum_{\lambda=1}^{g}{ }^{t} Q_{B_{\lambda}^{-1}}\left[X_{B_{\lambda} A_{\lambda} T_{\lambda-1}}-X_{A_{\lambda}{ }^{-1} B_{\lambda} A_{\lambda} T_{\lambda-1}}\right] \\
& +\sum_{\mu=1}^{j} Q_{C_{\mu}}\left[e_{\mu}^{-1} \sum_{m=1}^{e_{\mu}-1} X_{C_{\mu}^{m}}-X_{C_{\mu-1} \cdots C_{1} T_{8}}\right] \\
& -\sum_{\nu=1}^{k}{ }^{t} Q_{D_{\nu}}\left[X_{D_{\nu-1} \cdots D_{1} C_{j} \cdots C_{1} T_{8}}\right]+\sum_{\nu=1}^{k}{ }^{t} Q_{D_{\nu}} f\left(s_{\nu}\right) \text {. }
\end{aligned}
$$

For, let $t_{\mu}$ be a fixed point for $C_{\mu}(\mu=1, \cdots, j)$ in $\bar{\omega}_{0}$. Then

$$
f\left(t_{\mu}\right)=f\left(C_{\mu}^{m}\left(t_{\mu}\right)\right)=M\left(C_{\mu}^{m}\right) F\left(t_{\mu}\right)+X_{C_{\mu}^{m}}
$$

and

$$
e_{\mu} \mathfrak{F}\left(t_{\mu}\right)=\sum_{m=0}^{e_{\mu}-1} M\left(C_{\mu}^{m}\right) F\left(t_{\mu}\right)+\sum_{m=0}^{e_{\mu}-1} X_{C_{\mu}^{m}}
$$

On the other hand

$$
0=Q_{C_{\mu}^{e_{\mu}}}=\sum_{m=0}^{e_{\mu}-1}{ }^{t} M\left(C_{\mu}^{m}\right) Q_{C_{\mu}}
$$

Hence 


$$
\begin{aligned}
& \sum_{\mu}{ }^{t} Q_{C_{\mu}} f\left(t_{\mu}\right)=\sum_{\mu}{ }^{t} Q_{C_{\mu}} e^{-1}\left\{\sum_{m=0}^{e_{\mu}-1} M\left(C_{\mu}^{m}\right) f\left(t_{\mu}\right)+\sum_{m=0}^{e_{\mu-1}} x_{C_{\mu}^{m}}\right\} \\
& =\sum_{\mu} e_{\mu}^{-1}\left\{\sum_{m=0}^{e_{\mu}-1}{ }^{t} Q_{C_{\mu}}{ }^{M\left(C_{\mu}^{m}\right) f\left(t_{\mu}\right)}+{ }^{t} Q_{C_{\mu}} \sum_{m=0}^{e_{\mu-1}} x_{C_{\mu}^{m}}\right\} \\
& =\sum_{\mu}{ }^{t} Q_{C_{\mu}}\left(\sum_{m=0}^{e_{\mu}-1} e_{\mu}^{-1} X_{C_{\mu}}\right)+\sum_{\mu} e_{\mu}^{-1}\left(\sum_{m=0}^{e_{\mu}-1}{ }^{t} Q_{C_{\mu}}{ }^{M\left(C_{\mu}^{m}\right) \mathfrak{F}\left(t_{\mu}\right)}\right) \\
& =\sum_{\mu}{ }^{t} Q_{C_{\mu}} e_{\mu}^{-1}\left(\sum_{m=0}^{e^{\mu-1}} X_{C_{\mu}}\right) \text {. }
\end{aligned}
$$

We have

$$
\begin{aligned}
& \sum_{\mu}{ }^{t} Q_{C_{\mu}} \mathcal{F}\left(C_{\mu-1} \ldots C_{1} T_{B}\left(u_{0}\right)\right) \\
& \left.\quad=\sum_{\mu}{ }^{t} Q_{C_{\mu}}{ }^{M\left(C_{\mu-1}\right.} \cdots C_{1} T_{8}\right) \mathcal{F}\left(u_{0}\right)+\sum_{\mu}{ }^{t} Q_{C_{\mu}}{ }^{X} C_{\mu-1} \cdots C_{1} T_{g} .
\end{aligned}
$$

Furthermore we have

$$
\begin{aligned}
\sum_{\nu}{ }^{t} Q_{D_{\nu}}\left(f\left(s_{\nu}\right)-f\left(D_{\nu-1} \cdots D_{1} C_{j} \cdots C_{1} T_{g}\left(u_{0}\right)\right)\right. \\
\left.=\sum_{\nu}{ }^{t} Q_{D_{\nu}} f\left(s_{\nu}\right)-\sum_{\nu}{ }^{t} Q_{D_{\nu}}{ }^{M\left(D_{\nu-1}\right.} \cdots D_{1} C_{j} \cdots C_{1} T_{g}\right) f\left(u_{0}\right) \\
\quad-\sum_{\nu}{ }^{t} Q_{D_{\nu}} X_{D_{\nu-1} \cdots D_{1} C_{j} \cdots C_{1} T_{g}}
\end{aligned}
$$

We denote by $\Psi$ the sum of the first four terms of (9). Set

$$
\begin{aligned}
N_{1}= & \sum_{\lambda=1}^{g}{ }^{t}\left(M\left(A_{\lambda}^{-1} B_{\lambda} A_{\lambda} T_{\lambda-1}\right)-M\left(T_{\lambda-1}\right)\right) Q_{A_{\lambda}} \\
& +\sum_{\lambda=1}^{g}{ }^{t}\left(M\left(B_{\lambda} A_{\lambda} T_{\lambda-1}\right)-M\left(A_{\lambda}^{-1} B_{\lambda} A_{\lambda} T_{\lambda-1}\right)\right) Q_{B_{\lambda}^{-1}}, \\
N_{2}= & \sum_{\mu=1}^{j} t^{t} M\left(C_{\mu-1} \ldots C_{1} T_{8}\right) Q_{C_{\mu}} \text { and } \\
N_{3}= & \sum_{\nu=1}^{k}{ }^{t} M\left(D_{\nu-1} \ldots D_{1} C_{j} \ldots C_{1} T_{8}\right) Q_{D_{\nu}}
\end{aligned}
$$

Then we have 


$$
\begin{aligned}
& N_{1}=\sum_{\lambda=1}^{g}\left(Q_{T_{\lambda-1}}-Q_{T_{\lambda}}\right), \\
& N_{2}=\sum_{\mu=1}^{j}\left(Q_{C_{\mu} C_{\mu-1} \cdots C_{1} T_{g}}-Q_{C_{\mu-1} \cdots C_{1} T_{g}}\right), \text { and } \\
& N_{3}=\sum_{\nu=1}^{k}\left(Q_{D_{\nu} \cdots D_{1} C_{j} \cdots C_{1} T_{8}}-Q_{D_{\nu-1} \cdots D_{1} C_{j} \cdots C_{1} T_{g}}\right),
\end{aligned}
$$

hence $N_{1}-N_{2}-N_{3}=0$, since $D_{k} \cdots D_{1} C_{j} \cdots C_{1} T_{g}=1$, that is, $Q_{D_{k} \cdots D_{1} C_{j} \cdots C_{1} T_{g}}=0$. Thus we have the desired result. Our proof is now complete.

Corollary 1 (Period inequality). Under the same assumptions as in Theorem $1($ let $\phi=\psi)$,

$$
\begin{aligned}
& \frac{1}{2 i}\left\{\sum_{\lambda=1}^{g}{ }^{t} Q_{A_{\lambda}}\left[X_{A_{\lambda}{ }^{-1} B_{\lambda}{ }^{A}{ }_{\lambda} T_{\lambda-1}}-X_{T_{\lambda-1}}\right]+\sum_{\lambda=1}^{g}{ }^{t} Q_{B_{\lambda}^{-1}}\left[X_{B_{\lambda} A_{\lambda} T_{\lambda-1}}-X_{A_{\lambda}{ }^{-1} B_{\lambda} A_{\lambda} T_{\lambda-1}}\right]\right. \\
& +\sum_{\mu=1}^{j} Q_{C_{\mu}}\left[e_{\mu}^{-1} \sum_{m=1}^{e_{\mu}-1} x_{C_{\mu}^{m}}-x_{C_{\mu-1} \cdots C_{1} T_{g}}\right]
\end{aligned}
$$

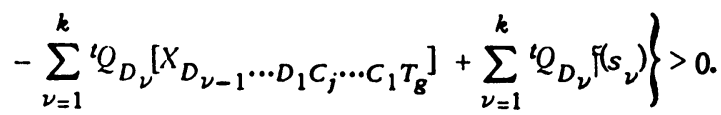

Corollary 2. Let $\Gamma$ be a fuchsian group of the first kind, and let $\Delta_{1}=U$. Let $f \in P E_{1-q}(U, \Gamma), f^{*} \in E_{1-q}(U, \Gamma), q \geq 2$, and $E, E^{*}$ arbitrary representatives of $f$ and $f^{*}$, respectively. Set $D^{2 q-1} E=\phi$ and $D^{2 q-1} E^{*}=\phi^{*}$. Let $f$ and $\mathrm{F}^{*}$ be column vectors of length $2 q-1$ of the form (1) associated with $E$ and $E^{*}$, respectively, and set $\operatorname{pd}_{A} f(z)=X_{A}$ and $\mathrm{pd}_{A} \mathrm{~F}^{*}(z)=X_{A}^{*}$ for each $A \epsilon$ $\Gamma$. Then

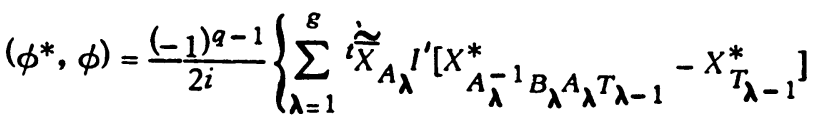

$$
\begin{aligned}
& +\sum_{\lambda=1}^{8}{ }^{t} \widetilde{\bar{X}}_{B_{\lambda}} I^{\prime}\left[X_{B_{\lambda} A_{\lambda} T_{\lambda-1}}^{*}-X_{A_{\lambda}^{-1} B_{\lambda} A_{\lambda} T_{\lambda-1}}^{*}\right] \\
& +\sum_{\mu=1}^{j} \widetilde{\widetilde{X}}_{C_{\mu}^{-1}} I^{\prime}\left[e_{\mu}^{-1} \sum_{m=1}^{e_{\mu}-1} X_{C_{\mu}^{m}}^{*}-X_{C_{\mu-1}}^{*} \cdots C_{1} T_{g}\right] \\
& \left.-\sum_{\nu-1}^{k}{ }^{\imath} \widetilde{\widetilde{X}}_{D_{\nu}^{-1}} I^{\prime}\left[X_{D_{\nu-1}}^{*} \cdots D_{1} c_{j} \cdots c_{1} T_{g}\right]+\sum_{\nu=1}^{k} \widetilde{\widetilde{X}}_{D_{\nu}^{-1}} I^{\prime} f\left(s_{\nu}\right)\right\} \text {. }
\end{aligned}
$$


Proof. If we set

$$
\psi(z)=\frac{(2 q-1) !}{2 \pi i} \iint_{U} \frac{(\zeta-\bar{\zeta} / 2 i)^{2 q-2} \overline{\phi(\zeta)} d \zeta \wedge d \zeta}{(\zeta-z)^{2 q}}, \quad z \in L,
$$

then $\psi \in B_{q}(L, \Gamma)$ (Bers [3]), where $L$ is the lower half-plane. Set $E_{1}(z)=$ $\operatorname{Pot}(\phi)(z), z \in L$. Then by Bers [3], $D^{2 q-1} E_{1}=\psi$ on $L$. If for $z \in U$, we set $E_{2}(z)=\overline{E_{1}(\bar{z})}$ and $D^{2 q-1} E_{2}(z)=\psi_{1}(z)$; then $\psi_{1}(z)=\overline{\psi(\bar{z}),} \psi_{1} \in B_{q}(U, \Gamma)$ and $E_{2} \in E_{1-q}(U, \Gamma)$. Then $\overline{\psi(\bar{z})}=c_{q} \phi(z), z \in U$ (see Kra [5, p. 554]), so that $\psi_{1}(z)=c_{q} \phi(z), z \in U$, where $c_{q}=(-1)^{q-1}(2 q-2) !$. Hence $E_{2}=c_{q} E$.

On the other hand if for each $A \in \Gamma$, we set $\operatorname{pd}_{A}(\operatorname{Pot}(\phi))=Q_{A}$ and $\operatorname{pd}_{A}(\mathcal{F})$ $=P_{A}$, then

$$
\begin{aligned}
E_{2}(A z) A^{\prime}(z)^{1-q}-E_{2}(z) & =\overline{E_{1}(\overline{A z}) A^{\prime}(\bar{z})^{1-q}-E_{1}(\bar{z})} \\
& =\frac{1}{n !} \bar{Q}_{A}\left(\begin{array}{l}
z \\
1
\end{array}\right)^{n}, \quad z \in U,
\end{aligned}
$$

and

$$
E(A z) A^{\prime}(z)^{1-q}-E(z)=\frac{1}{n !}{ }^{t} P_{A}\left(\begin{array}{c}
z \\
1
\end{array}\right)^{n}, \quad z \in U,
$$

where $\mathfrak{F}(z)=I^{\circ} \mathfrak{F ( z )}$. Hence $\bar{Q}_{A}=c_{q} P_{A}$. Using Lemma 3, we have

$$
\begin{aligned}
{ }^{t} P_{A} & \left.={ }^{t}\left(I^{\prime} \overparen{M(A-1)}\right) \tilde{X}_{A}\right) \\
& ={ }^{t} \tilde{X}_{A} \widetilde{t}_{M(A)}^{-1} I^{\prime} \\
& =-{ }^{t} \tilde{X}_{A-1} I^{\prime}
\end{aligned}
$$

Substituting these into (I), we have the desired result. Our proof is now complete.

Remark. By using the same method as in the proof of Theorem 2 below, we can show that even in the case of $q=1$, the above corollary remains valid.

4. Period relations and inequalities. By modifying Shimura's method [7], we can prove the following theorem from Lemmas 1, 2,3 and 4. The proof is omitted here.

Theorem 2 (Period relation). Let $\Gamma$ be the same group as in Theorem 1. Let $f_{1}, f_{2} \in E_{i-q}(\Delta, \Gamma, M), q \geq 1$ and $\mathfrak{F}_{1}, \mathfrak{F}_{2}$ arbitrary representatives of $f_{1}$ and $f_{2}$, respectively. Set $\mathrm{pd}_{A} \mathfrak{F}_{1}=P_{A}^{(1)}$ and $\mathrm{pd}_{A} \mathfrak{F}_{2}=P_{A}^{(2)}$ for each $A \in \Gamma$, Then 


$$
\begin{aligned}
& \sum_{\lambda=1}^{8}{ }^{t} P_{A_{\lambda}}^{(1)}\left[M\left(A_{\lambda}^{-1} B_{\lambda} A_{\lambda} T_{\lambda-1}\right) I^{\prime-1} \tilde{P}_{A_{\lambda}^{-1} B_{\lambda} A_{\lambda} T_{\lambda-1}}^{(2)}-M\left(T_{\lambda-1}\right) I^{\prime-1} \tilde{P}_{T_{\lambda-1}^{(2)}}^{(2)}\right] \\
& +\sum_{\lambda=1}^{8}{ }^{t} P_{B_{\lambda}^{-1}}^{(1)}\left[M\left(B_{\lambda} A_{\lambda} T_{\lambda-1}\right) l^{\prime}-1 \tilde{P}_{B_{\lambda}{ }^{A}}^{(2)} T_{\lambda-1}-M\left(A_{\lambda}^{-1} B_{\lambda} A_{\lambda} T_{\lambda-1}\right) I^{\prime-1} \tilde{P}_{A_{\lambda}^{-1}}^{(2)} B_{\lambda} A_{\lambda} T_{\lambda-1}\right] \\
& +\sum_{\mu=1}^{j} P_{C_{\mu}}^{(1)}\left[e_{\mu}^{-1} \sum_{m=1}^{e_{\mu-1}} M\left(C_{\mu}^{m}\right) I^{\prime-1} \tilde{P}_{e_{\mu}^{(2)}}^{(2)}-M\left(C_{\mu-1} \cdots C_{1} T_{8}\right) I^{\prime-1} \tilde{P}_{C_{\mu-1}^{(2)}} \cdots C_{1} T_{8}\right] \\
& -\sum_{\nu=1}^{k}{ }^{t} P_{D_{\nu}}^{(1)}\left[M\left(D_{\nu-1} \cdots D_{1} C_{j} \cdots C_{1} T_{8}\right) I^{\prime-1} \tilde{P}_{D_{\nu-1}}^{(2)} \cdots D_{1} C_{j} \cdots C_{1} T_{g}\right] \\
& +\sum_{\nu=1}^{k}{ }^{t} P_{D}^{(1)} f\left(s_{\nu}\right)=0
\end{aligned}
$$

Corollary 1 (Period relation for a fuchsian group). Let $\Gamma$ be a fuchsian group of the first kind, and let $\Delta_{1}=U$. Let $f_{1}, f_{2} \in E_{1-q}(\Lambda, \Gamma, M), q \geq 1$, and $\mathfrak{F}_{1}, \mathfrak{F}_{2}$ arbitrary representatives of $f_{1}$ and $f_{2}$, respectively and set $\mathfrak{F}_{1}=I^{\prime} \mathrm{F}_{1}$ and $\mathfrak{F}_{2}=$ $I^{\prime} \tilde{F}_{2}$. Set $\operatorname{pd}_{A} F_{1}=X_{A}^{(1)}$ and $\operatorname{pd}_{A} F_{2}=X_{A}^{(2)}$ for every $A \in \Gamma$. Then

$$
\begin{aligned}
& \sum_{\lambda=1}^{g} \tilde{x}_{A_{\lambda}^{(1)}}^{(1)} I^{\prime}\left[X_{A_{\lambda}^{(2)} B_{\lambda} A_{\lambda} T_{\lambda-1}}-X_{T_{\lambda-1}^{(2)}}^{(2)}\right] \\
& +\sum_{\lambda=1}^{8} \tilde{t}_{X_{B_{\lambda}}^{(1)}}^{\left(1 I^{\prime}\right.}\left[X_{B_{\lambda} A_{\lambda} T_{\lambda-1}^{(2)}}-X_{\left.A_{\lambda}^{-1} B_{\lambda} A_{\lambda} T_{\lambda-1}\right]}\right] \\
& +\sum_{\mu=1}^{j} \tilde{t}_{C_{\mu}^{(1)}}^{(1)} I^{\prime}\left[e_{\mu}^{-1} \sum_{m=1}^{e_{\mu-1}} X_{C_{\mu}^{(2)}}^{(2)}-X_{C_{\mu-1}^{(2)}} \cdots C_{1} T_{g}\right]
\end{aligned}
$$

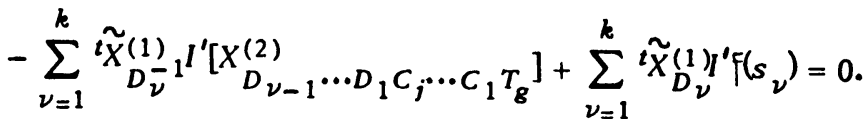

Remark. Especially, when $q=1$,

$$
\sum_{\lambda=1}^{8}\left(X_{B_{\lambda}}^{(1)} X_{A_{\lambda}}^{(2)}-X_{A_{\lambda}}^{(1)} X_{B_{\lambda}}^{(2)}\right)=0 .
$$

For $I^{\prime}=1$ and $X_{A-1}^{(i)}=-X_{A}^{(i)}(i=1,2)$ for all $A \in \Gamma$. This is the period relation for abelian integrals. We set $X_{A}=x_{A}+i y_{A}$, where $x_{A}$ and $y_{A}$ are real number vectors.

Corollary 2 (Period inequality). Let $\Gamma$ be the same group as in the above corollary. Let $f \in E_{1-q}(U, \Gamma, M)$ and $₹$ be representative of $f, q \geq 1$. Set 
$\mathfrak{F}=I^{\circ} \mathfrak{F},(1 / n !) D^{2 q-1} \mathfrak{F}(z)\left(z_{1}^{z}\right)^{n}=\phi(z)$ and $\mathrm{pd}_{A} \mathrm{~F}(z)=x_{A}+i y_{A}$ for every $A \in \Gamma$. If $\phi \neq 0$, then

$$
\begin{aligned}
& (-1)^{q}\left[\sum_{\lambda=1}^{B}{ }^{2} \tilde{y}_{A_{\lambda}-{ }^{-1}} l^{\prime}\left(x_{A_{\lambda}}{ }^{-1} B_{\lambda} A_{\lambda} T_{\lambda-1}-x T_{\lambda-1}\right)\right. \\
& +\sum_{\lambda=1}^{g}{ }^{\imath} \tilde{y}_{B_{\lambda}} l^{\prime}\left(x_{B_{\lambda} A_{\lambda} T_{\lambda-1}}-x_{A_{\lambda}{ }^{-1} B_{\lambda} A_{\lambda} T_{\lambda-1}}\right)+\sum_{\mu=1}^{j}{ }^{\imath} \tilde{y}_{C_{\mu}^{-1}}{ }^{\prime}\left[e_{\mu}^{-1} \sum_{m=1}^{e^{\mu-1}} x_{C_{\mu}^{m}}-x_{C_{\mu-1} \cdots C_{1}} T_{g}\right] \\
& \left.-\sum_{\nu=1}^{k} \tilde{y}_{D_{\nu}^{-1}} I^{\prime}\left[x_{D_{\nu-1}} \cdots D_{1} C_{i} \cdots C_{1} T_{g}\right]+\sum_{\nu=1}^{k}{ }^{i} \tilde{y}_{D_{\nu}^{-1}} l^{\prime}\left(\operatorname{Re} f\left(s_{\nu}\right)\right)\right]>0 \text {. }
\end{aligned}
$$

Proof. Set

$$
\begin{aligned}
& \Phi_{1}=\sum_{\lambda=1}^{8}{ }^{i} \tilde{x}_{A_{\lambda}{ }^{-1}} I^{\prime}\left[x_{A_{\lambda}{ }^{-1} B_{\lambda} A_{\lambda} T_{\lambda-1}}-x_{T_{\lambda-1}}\right]+\sum_{\lambda=1}^{8}{ }^{i} \widetilde{x}_{B_{\lambda}} I^{\prime}\left[x_{B_{\lambda} A_{\lambda} T_{\lambda-1}}-x_{A_{\lambda}{ }^{-1} B_{\lambda} A_{\lambda} T_{\lambda-1}}\right] \\
& +\sum_{\mu=1}^{i} \tilde{x}_{C_{\mu}^{-1}} I^{\prime}\left[e_{\mu}^{-1} \sum_{m=1}^{e \mu-1} x_{C_{\mu}^{m}}-x_{C_{\mu-1}} \cdots C_{1} T_{g}\right]-\sum_{\nu=1}^{k}{ }^{i} \tilde{x}_{D_{\nu}^{-1}} l^{\prime}\left[x_{D_{\nu-1}} \cdots D_{1} C_{j} \cdots C_{1} T_{g}\right] \\
& +\sum_{\nu=1}^{k}{ }^{i} \tilde{x}_{D_{\nu}{ }^{-1}} l^{\prime} \operatorname{Re} f\left(s_{\nu}\right) \text {, }
\end{aligned}
$$

$$
\begin{aligned}
& \Phi_{2}=\sum_{\lambda=1}^{8}{ }^{t} \tilde{x}_{A_{\lambda}-{ }^{-1}} I^{\prime}\left[y_{A_{\lambda}{ }^{-1} B_{\lambda} A_{\lambda} T_{\lambda-1}}-y_{T_{\lambda-1}}\right]+\sum_{\lambda=1}^{E}{ }^{t} \tilde{x}_{B_{\lambda}} I^{\prime}\left[y_{B_{\lambda} A_{\lambda} T_{\lambda-1}}-y_{\left.A_{\lambda}{ }^{-1} B_{\lambda} A_{\lambda} T_{\lambda-1}\right]}\right] \\
& +\sum_{\mu=1}^{i}{ }^{i} \tilde{x}_{C_{\mu}^{-1}} I^{\prime}\left[e_{\mu}^{-1} \sum_{m=1}^{e \mu-1} y_{C_{\mu}^{m}}-y_{C_{\mu-1} \cdots C_{1} T_{g}}\right]-\sum_{\nu=1}^{k}{ }^{i} \tilde{x}_{D_{\nu}{ }^{-1}} l^{\prime}\left[y_{D_{\nu-1}} \cdots D_{1} C_{j} \cdots \varepsilon_{1} T_{g}\right] \\
& +\sum_{\nu=1}^{k}{ }^{t} x_{D_{\nu}-l^{-1}}{ }^{\prime} \operatorname{Im} f\left(s_{\nu}\right) \text {, }
\end{aligned}
$$

$$
\begin{aligned}
& \Phi_{3}=\sum_{\lambda=1}^{8}{ }^{t} \tilde{y}_{A_{\lambda}{ }^{-1}}{ }^{\prime}\left[x_{A_{\lambda}{ }^{-1} B_{\lambda} A_{\lambda} T_{\lambda-1}}-x_{T_{\lambda-1}}\right]+\sum_{\lambda=1}^{R}{ }^{t} \tilde{y}_{B_{\lambda}} I^{\prime}\left[x_{B_{\lambda} A_{\lambda} T_{\lambda-1}}-x_{A_{\lambda}{ }^{-1} B_{\lambda} A_{\lambda} T_{\lambda-1}}\right] \\
& +\sum_{\mu=1}^{i} \tilde{y}_{C_{\mu}^{-1}} l^{\prime}\left[e_{\mu}^{-1} \sum_{m=1}^{e} x_{C_{\mu}^{m}}-x_{C_{\mu-1}} \cdots C_{1} T_{g}\right]-\sum_{\nu=1}^{k} \tilde{y}_{D_{\nu}^{-1}} l^{\prime}\left[x_{D_{\nu-1}} \cdots D_{1} C_{j} \cdots C_{1} T_{g}\right] \\
& +\sum_{\nu=1}^{k} \tilde{y}_{D_{\nu}^{-1}} I^{\prime} \operatorname{Ref}\left(s_{\nu}\right) \text {, }
\end{aligned}
$$




$$
\begin{aligned}
& \Phi_{4}=\sum_{\lambda=1}^{8}{ }^{2} \tilde{y}_{A_{\lambda}{ }^{-1}} I^{\prime}\left[y_{A_{\lambda}{ }^{-1} B_{\lambda} A_{\lambda} T_{\lambda-1}}-y_{T_{\lambda-1}}\right]+\sum_{\lambda=1}^{8}{ }^{i} \tilde{y}_{B_{\lambda}} I^{\prime}\left[y_{B_{\lambda} A_{\lambda} T_{\lambda-1}}-y_{A_{\lambda}{ }^{-1} B_{\lambda} A_{\lambda} T_{\lambda-1}}\right] \\
& +\sum_{\mu=1}^{j}{ }^{i} \tilde{y}_{C_{\mu}^{-1}} l^{\prime}\left[e_{\mu}^{-1} \sum_{m=1}^{e_{\mu}^{-1}} y_{C_{\mu}^{m}}-y_{C_{\mu-1}} \cdots C_{1} T_{g}\right]-\sum_{\nu=1}^{k}{ }^{2} \tilde{y}_{D_{\nu}^{-1}} l^{\prime}\left[y_{D_{\nu-1}} \cdots D_{1} C_{j} \cdots C_{1} T_{g}\right] \\
& +\sum_{\nu=1}^{k}{ }^{t} \tilde{y}_{D_{\nu}^{-1}} I^{\prime} \operatorname{Im} f\left(s_{\nu}\right) \text {. }
\end{aligned}
$$

Combining the equations (II) and (III) in the case of $\phi=\psi \neq 0$, we have

$$
\Phi_{1}+i \Phi_{2}-i \Phi_{3}+\Phi_{4}=2 i(-1)^{q-1}\|\phi\|^{2}
$$

and

$$
\Phi_{1}+i \Phi_{2}+i \Phi_{3}-\Phi_{4}=0
$$

Thus $\Phi_{1}=\Phi_{4}=0$ and $\Phi_{3}=-\Phi_{2}=(-1)^{q}\|\phi\|^{2}$. Since $(-1)^{q} \Phi_{3}>0$, we have the desired result.

Remark. Especially, when $q=1$,

$$
\text { (-1) } \sum_{\lambda=1}^{8}\left(y_{B_{\lambda}}{ }^{x} A_{\lambda}-y_{A_{\lambda}}{ }^{x} B_{\lambda}\right)>0 \text {. }
$$

This is the period inequality for abelian integrals.

The following result of $\mathrm{Kra}[5]$ is obtained from the above corollary.

Corollary 3. Let $\Gamma$ be the same group as in the above corollary. If $X_{A}$ is real for every $A \in \Gamma$, then $X_{A}=0$.

Proof. In Corollary 2 to Theorem 2, we have $y_{A_{\lambda}}=0, y_{B_{\lambda}}=0(\lambda=1, \cdots$, $g), y_{C_{\mu}}=0(\mu=1, \cdots, j)$ and $y_{D_{\nu}}=0(\nu=1, \cdots, k)$, and so $\phi$ must be zero. Hence $X_{A}=0$.

Finally we consider meromorphic Eichler integrals. We denote by $M_{1-q}(\Delta, \Gamma)$ the space of identified meromorphic Eichler integrals. Then we have the following:

Theorem 3. Let $\Gamma$ be the same group as in Theorem 1. Assume, for the sake of simplicity, that the group bas neitber parabolic nor elliptic elements. Let $f \in M_{1-q}(\Delta, \Gamma), q \geq 1$, and $E$ an arbitrary representative of $f$. Let $E$ bave only one pole at $u_{1}$ in $\omega_{0}$ with principal part $\left(1 / z^{m}\right), m \geq 1$. Let $E^{*}$ be an arbitrary representative of $f^{*}, f^{*} \in E_{1-q}(\Delta, \Gamma)$ such that $D^{2 q-1} E^{*}=\phi^{*} \in B_{q}(\Delta, \Gamma)$ bas the representation $\phi^{*}(z)=\left(c_{0}+c_{1} z+\cdots\right) d z^{q}$ about $u_{1}$. Let $\operatorname{pd}_{A}^{q} F=X_{A}$ and 
$\mathrm{pd}_{A} \mathrm{~F}^{*}=X_{A}^{*}$ for eacb $A \in \Gamma$, where $F$ and $\mathrm{F}^{*}$ are column vectors of length $2 q-$ 1 of the form (1) associated with $E$ and $E^{*}$, respectively. Then

$$
\begin{aligned}
\sum_{\lambda=1}^{g}\left[\left({ }^{t} \tilde{X}_{A_{\lambda}{ }^{-1}} I^{\prime} X_{B_{\lambda}}^{*}-{ }^{t} \tilde{X}_{B_{\lambda}{ }^{-1}} I^{\prime} X_{A_{\lambda}}^{*}\right)\right. & +{ }^{t}\left(\tilde{X}_{A_{\lambda}}-\tilde{X}_{B_{\lambda}^{-1}}\right) l^{\prime} M\left(A_{\lambda}\right) X_{T_{\lambda-1}^{*}}^{*} \\
& \left.t\left(\tilde{X}_{A_{\lambda}^{-1}}-\tilde{X}_{B_{\lambda}}\right) I^{\prime} M\left(B_{\lambda}\right) X_{T_{\lambda}}^{*}\right]-2 \pi i n ! c_{m-1}
\end{aligned}
$$

Proof. We easily see that

$$
\int t^{t} \mathcal{F}(z) I^{\prime}\left(\begin{array}{l}
1 \\
z
\end{array}\right)^{n} \phi^{*}(z) d z=n ! \int E(z) \phi^{*}(z) d z=2 \pi i n ! c_{m-1} \text {. }
$$

By using the same way as in Theorem 2, we see that the left-side hand is equal to

$$
\begin{aligned}
\sum_{\lambda=1}^{R}\left[\left(\tilde{X}_{A_{\lambda}^{-1}} I^{\prime} X_{B_{\lambda}}^{*}-{ }^{t} \tilde{X}_{B_{\lambda}^{-1}} I^{\prime} X_{A_{\lambda}}^{*}\right)+\left({ }^{t}\left(\tilde{X}_{A_{\lambda}}-\tilde{X}_{B_{\lambda}^{-1}}\right) I^{\prime} M\left(A_{\lambda}\right) X_{T_{\lambda-1}}^{*}\right)\right. & \\
& \left.+{ }^{t}\left(\tilde{X}_{A_{\lambda}^{-1}}-\tilde{X}_{B_{\lambda}}\right) I^{\prime} M\left(B_{\lambda}\right) X_{T_{\lambda}}^{*}\right]
\end{aligned}
$$

Remark. Let $\tilde{\Delta}$ be a finite sum of nonequivalent simply connected components $\Delta_{1}, \Delta_{2}, \cdots, \Delta_{t}$, that is, $\tilde{\Delta}=\bigcup_{i=1}^{t} \Delta_{i}$, where $\Delta_{i} \neq A\left(\Delta_{j}\right)(i \neq j)$ for any $A$ $\epsilon \Gamma$. Then we obta in similar results as above.

\section{REFERENCES}

1. L. V. Ahlfors, Finitely generated Kleinian groups, Amer. J. Math. 86 (1964), 413429; 87 (1965), 759. MR 29 \#4890; MR 31 \#4906.

2. - The structure of a finitely generated Kleiniain group, Acta Math. 122 (1969), 1-17. MR $38 \# 6063$.

3. L. Bers, Inequalities for finitely generated Kleinian groups, J. Anal. Math. 18 (1967), 23-41. MR 37 \#5383.

4. M. Eichler, Eine Verallgemeinerung der Abelschen Integrale, Math. Z. 67 (1957), 267-298. MR 19, 740.

5. I. Kra, On cohomology of kleinian groups, Ann. of Math. (2) 89 (1969), 533-556. MR 41 \#8656a.

6. - On cohomology of kleinian groups. II, An n. of Math. (2) 90 (1969), 576590. MR $41 \# 8656$ b.

7. G. Shimura, Sur les intégrales attachées aux formes automorphes, J. Math. Soc. Japan 11 (1959), 291-311. MR 22 \#11126.

DEPARTMENT OF MATHEMATICS, SHIZUOKA UNIVERSITY, OHYA SHIZUOKA, JAPAN 